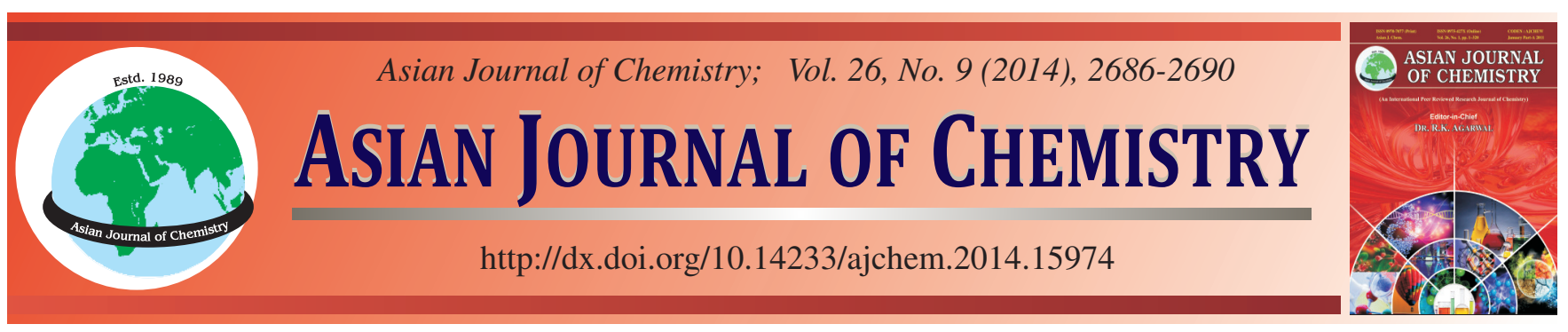

\title{
Synthesis of Caffeic Acid Phenethyl Ester Analogues and Their Cytotoxicities Against Human Cancer Cells
}

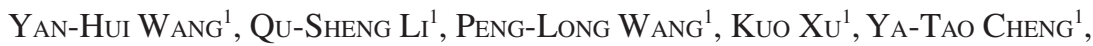 \\ XIN Xu' ${ }^{1}$, Qiang Li ${ }^{1}$, Yu-Zhong Zhang ${ }^{2}$ and Hai-Min LeI ${ }^{1, *}$
}

${ }^{1}$ School of Chinese Pharmacy, Beijing University of Chinese Medicine, Beijing 100102, P.R. China

${ }^{2}$ School of Basic Medical Sciences, Beijing University of Chinese Medicine, Beijing 100102, P.R. China

*Corresponding author: Tel: +86 108473 8640,E-mail: hm_lei@126.com

\begin{abstract}
Although caffeic acid phenethyl ester exhibits strong antitumor property, but its pharmacophore has not been elaborated clearly as yet. Seventeen caffeic acid phenethyl ester analogues were synthesized via simple and easy procedures and their antiproliferative effects were evaluated toward Bel-7402 and MCF-7 cancer cell lines. Compared to compounds 1-11, 12, 13, 14, 15 showed cytotoxic effects. The IC $\mathrm{C}_{50}$ values $(\mu \mathrm{M})$ of compound $14(41.87,51.98)$ were about equal to caffeic acid phenethyl ester's $(34.60,42.26)$. Structure-activity relationship analysis demonstrated that the $\alpha, \beta$-unsaturated double bond and the phenolic hydroxyl group in caffeic acid phenethyl ester were essential for the antitumor activities. Besides, the cytotoxic properties of these compounds were determined by the methoxy groups on the phenyl ring and the location of aromatic hydroxyl groups. These results may be of great benefit to people in screening new anticancer drug candidates.
\end{abstract}

Keywords: Caffeic acid phenethyl ester analogues, Pharmacophore, Cytotoxic properties.

\section{INTRODUCTION}

Caffeic acid phenethyl ester (CAPE, Fig. 1) is a natural ingredient extracted from propolis ${ }^{1}$. It is known to exhibit antiinflammatory, antioxidant, antineoplastic and immunomodulatory properties ${ }^{2-5}$. In particular, its anticancer effects have been highly investigated, which have been demonstrated in a number of cancer models, such as breast cancer, hepatocellular cancer, oral cancer, cholangio carcinoma, human melanoma, glioblastoma multiforme, prostate cancer and lung cancer ${ }^{6-12}$. Caffeic acid phenethyl ester shows strong anticancer effect via its apoptotic effects and modulation of $\mathrm{NF}-\mathrm{KB}^{6,11}$.<smiles>O=C(/C=C/c1ccc(O)c(O)c1)OCCc1ccccc1</smiles>

Because of CAPE's simplicity of structure and interesting cytotoxic property, several researchers have started to explore strategies for the synthesis of CAPE or CAPE analogues and have studied their activities ${ }^{13-15}$. However, there is scant literature to illustrate CAPE's structure-activity relationship.
To find the pharmacophore, we designed and synthesized seventeen caffeic acid phenethyl ester analogues (Scheme-I), which are easily obtained. We tested their in vitro antitumor activities by 3-(4,5-dimethyl-2-thiazolyl)-2,5-diphenyl tetrazolium bromide (MTT) assay on hepatocellular cancer cell lines (Bel-7402) and human breast cancer cell lines (MCF-7).

\section{EXPERIMENTAL}

All commercially available solvents and reagents were used without further purification. Melting points were determined with a BÜCHI Melting Point B-450 apparatus (Büchi Labortechnik, Flawil, Switzerland). The mass spectra were obtained by using an ion trap-mass spectrometer (3200 QTRAP; Applied Biosystems, Foster City, California, USA), the ${ }^{1} \mathrm{H}$ NMR signals were obtained using a $500 \mathrm{MHz}$ Bruker (Bruker Corporation, Billerica, Massachusetts, USA), ${ }^{13} \mathrm{C}$ NMR were obtained in a Mercury-200 at $125 \mathrm{MHz}$ (Varian Inc., Palo Alto, California, USA). All the reactions were monitored by thinlayer chromatography (TLC) on precoated silica gel G plates, which were observed at $254 \mathrm{~nm}$ under a UV lamp. TLC was performed using precoated silica gel GF254 $(0.2 \mathrm{~mm}$; Chemical Industry Institute, Yantai, China) and column chromatography was performed using silica gel (100-200 mesh; Haiyang Chemical Co., Ltd, Qingdao, China). Caffeic acid phenethyl ester was 


\begin{tabular}{ccccc} 
& & & \\
\hline
\end{tabular}

Scheme-I: The structure of compounds 1-17 and synthetic route of caffeic acid phenethyl ester analogues

purchased from Sigma Chemical Co. (St Louis, Missouri, USA).

Fetal bovine serum (FBS) was obtained from Gibco. Penicillin and streptomycin were obtained from Thermo Scientific. Hepatocellular cancer cell lines and human breast cancer cell ines were obtained from the Institute of Medicine of Peking Union of Beijing.

Synthesis

3-Phenyl-acrylic acid phenethyl ester (1): A solution of 2-phenyl-ethanol $(0.989 \mathrm{~g}, 8.10 \mathrm{mmol})$ in $25 \mathrm{~mL}$ of $\mathrm{CH}_{2} \mathrm{Cl}_{2}$ was stirred at room temperature. EDCI $(0.989 \mathrm{~g})$ and DMAP $(0.329 \mathrm{~g})$ were added into the solution while stirring. After $0.5 \mathrm{~h}, 3$-phenyl-acrylic acid (1.000 g, $6.76 \mathrm{mmol})$ was added to the reaction mixture and allowed to stay overnight. The solution was evaporated to a small volume, then purified by column chromatography (silica gel). Elution with petroleumacetone $(30: 1, \mathrm{v} / \mathrm{v})$ produced pure compound $\mathbf{1}$. White solid, yield $92 \%$. m.p.: $54.3-55.3{ }^{\circ} \mathrm{C} ;{ }^{1} \mathrm{H} \mathrm{NMR}\left(500 \mathrm{MHz}, \mathrm{CDCl}_{3}\right) \delta$ $7.71(\mathrm{~d}, J=16.0 \mathrm{~Hz}, 1 \mathrm{H}), 7.58-7.54(\mathrm{~m}, 2 \mathrm{H}), 7.44-7.24(\mathrm{~m}$, $8 \mathrm{H}), 6.46(\mathrm{~d}, J=16.0 \mathrm{~Hz}, 1 \mathrm{H}), 4.46(\mathrm{t}, J=7.1 \mathrm{~Hz}, 2 \mathrm{H}), 3.06(\mathrm{t}$, $J=7.0 \mathrm{~Hz}, 2 \mathrm{H}) ;{ }^{13} \mathrm{C}$ NMR $\left(126 \mathrm{MHz}, \mathrm{CDCl}_{3}\right) \delta 166.90(\mathrm{C}-1)$, 144.87 (C-3), 137.91 (C-3'), 134.44 (C-4), 130.30 (C-5', C-7') 128.96 (C-6, C-8), 128.90 (C-4', C-8'), 128.54 (C-7), 128.10 (C-5, C-9), 126.60 (C-6'), 118.09 (C-2), 65.04 (C-1'), 35.24 (C-2'); MS (ESI) m/z: $253[\mathrm{M}+\mathrm{H}]^{+}$, calcd. for $\mathrm{C}_{17} \mathrm{H}_{16} \mathrm{O}_{2} 252.12$.
Compounds 2-11 were prepared in the same manner as described for compound $\mathbf{1}$.

3-(3,4-Dimethoxy-phenyl)acrylic acid phenethyl ester (2): White solid, yield $90 \%$, m.p.: 101.2-102.1 ${ }^{\circ} \mathrm{C} ;{ }^{1} \mathrm{H}$ NMR $\left(500 \mathrm{MHz}, \mathrm{CDCl}_{3}\right) \delta 7.65(\mathrm{~d}, J=15.9 \mathrm{~Hz}, 1 \mathrm{H}), 7.35(\mathrm{t}, J=7.4$ $\mathrm{Hz}, 2 \mathrm{H}), 7.28$ (dd, $J=15.2,7.6 \mathrm{~Hz}, 3 \mathrm{H}), 7.10$ (dd, $J=25.5$, $4.9 \mathrm{~Hz}, 2 \mathrm{H}), 6.88$ (t, $J=9.8 \mathrm{~Hz}, 1 \mathrm{H}), 6.33(\mathrm{~d}, J=15.9 \mathrm{~Hz}$, $1 \mathrm{H}), 4.45(\mathrm{t}, J=7.1 \mathrm{~Hz}, 2 \mathrm{H}), 3.94(\mathrm{~s}, 6 \mathrm{H}), 3.05(\mathrm{t}, J=7.0 \mathrm{~Hz}$, $2 \mathrm{H}) ;{ }^{13} \mathrm{C} \mathrm{NMR}\left(126 \mathrm{MHz}, \mathrm{CDCl}_{3}\right) \delta 167.13(\mathrm{C}-1), 151.16(\mathrm{C}-$ 6), 149.24 (C-7), 144.79 (C-3), 137.96 (C-3'), 128.93 (C-5', C-7'), 128.53 (C-4), 127.42 (C-4', C-8'), 126.56 (C-6'), 122.67 (C-9), 115.75 (C-8), 111.05 (C-2), 109.64 (C-5), 64.88 (C$\left.1^{\prime}\right), 55.99\left(\mathrm{C}-3, \mathrm{OCH}_{3}\right), 55.91\left(\mathrm{C}-4, \mathrm{OCH}_{3}\right), 35.26\left(\mathrm{C}-2^{\prime}\right)$; $\mathrm{MS}$ (ESI) $m / z: 313[\mathrm{M}+\mathrm{H}]^{+}$, calcd. for $\mathrm{C}_{19} \mathrm{H}_{20} \mathrm{O}_{4} 312.14$.

3-(3,4,5-Trimethoxy-phenyl)acrylic acid phenethyl ester (3): White solid, yield $92 \%$, m.p.: $98.7-99.8{ }^{\circ} \mathrm{C} ;{ }^{1} \mathrm{H}$ NMR $\left(500 \mathrm{MHz}, \mathrm{DMSO}_{-} \mathrm{d}_{6}\right) \delta 7.62(\mathrm{~d}, J=15.9 \mathrm{~Hz}, 1 \mathrm{H}), 7.39-7.25$ $(\mathrm{m}, 5 \mathrm{H}), 6.77(\mathrm{~s}, 2 \mathrm{H}), 6.36(\mathrm{~d}, J=15.9 \mathrm{~Hz}, 1 \mathrm{H}), 4.46(\mathrm{t}, J=$ $7.0 \mathrm{~Hz}, 2 \mathrm{H}), 3.90$ (t, $J=6.6 \mathrm{~Hz}, 9 \mathrm{H}), 3.05(\mathrm{t}, J=7.0 \mathrm{~Hz}, 2 \mathrm{H})$. ${ }^{13} \mathrm{C}$ NMR (126 MHz, DMSO-d $\left.{ }_{6}\right) \delta 166.84(\mathrm{C}-1), 153.47$ (C-6, C-8), 144.84 (C-3), 137.88 (C-3'), 129.90 (C-7), 128.91 (C-4), 128.54 (C-5', C-7'), 126.58 (C-4', C-8'), 117.29 (C-2), 105.35 (C-5, C-9), 64.97 (C-1'), $60.96\left(\mathrm{C}-7, \mathrm{OCH}_{3}\right), 56.19$ (C-6, $\left.\mathrm{OCH}_{3} ; \mathrm{C}-8, \mathrm{OCH}_{3}\right), 35.23(\mathrm{C}-2$ '); MS (ESI) m/z: 343 $[\mathrm{M}+\mathrm{H}]^{+}$, calcd. for $\mathrm{C}_{20} \mathrm{H}_{22} \mathrm{O}_{5} 342.15$.

3-(4-Methoxy-phenyl)acrylic acid phenethyl ester (4): White solid, yield $95 \%$, m.p.: 56.7-57.6 ${ }^{\circ} \mathrm{C}$; ${ }^{1} \mathrm{H}$ NMR (500 MHz, DMSO-d $\left.{ }_{6}\right) \delta 7.66(\mathrm{~d}, J=16.0 \mathrm{~Hz}, 1 \mathrm{H}), 7.50(\mathrm{~d}, J=8.6$ $\mathrm{Hz}, 2 \mathrm{H}), 7.38-7.24$ (m, 5H), 6.93 (d, $J=8.6 \mathrm{~Hz}, 2 \mathrm{H}), 6.33$ (d, $J=16.0 \mathrm{~Hz}, 1 \mathrm{H}), 4.45(\mathrm{t}, J=7.1 \mathrm{~Hz}, 2 \mathrm{H}), 3.87(\mathrm{~s}, 3 \mathrm{H}), 3.05(\mathrm{t}$, $J=7.0 \mathrm{~Hz}, 2 \mathrm{H}) .{ }^{13} \mathrm{C} \mathrm{NMR}\left(126 \mathrm{MHz}, \mathrm{CDCl}_{3}\right) \delta 167.24(\mathrm{C}-1)$, 161.41 (C-7), 144.53 (C-3), 137.99 (C-3'), 129.76 (C-5', C7'), 128.96 (C-4', C-8'), 128.52 (C-5,C-9), 127.17 (C-4), 126.56 (C-6'), 115.55 (C-2), 114.34 (C-6, C-8), 64.88 (C-1'), 55.39 $\left(\mathrm{C}-7,-\mathrm{OCH}_{3}\right), 35.27\left(\mathrm{C}-2^{\prime}\right)$; MS (ESI) $m / z: 283[\mathrm{M}+\mathrm{H}]^{+}$, calcd. for $\mathrm{C}_{18} \mathrm{H}_{18} \mathrm{O}_{3} 282.13$.

3-(2, 3-Dimethoxy-phenyl)-acrylic acid phenethyl ester (5): White solid, yield $95 \%$, m.p.: 38.3-39.2 ${ }^{\circ} \mathrm{C}$; ${ }^{1} \mathrm{H}$ NMR $\left(500 \mathrm{MHz}, \mathrm{CDCl}_{3}\right) \mathrm{d} 8.02(\mathrm{~d}, J=16.2 \mathrm{~Hz}, 1 \mathrm{H}), 7.37-7.26(\mathrm{~m}$, $5 \mathrm{H}), 7.18(\mathrm{~d}, J=7.9 \mathrm{~Hz}, 1 \mathrm{H}), 7.09(\mathrm{t}, J=8.0 \mathrm{~Hz}, 1 \mathrm{H}), 6.97(\mathrm{~d}$, $J=8.0 \mathrm{~Hz}, 1 \mathrm{H}), 6.49(\mathrm{~d}, J=16.2 \mathrm{~Hz}, 1 \mathrm{H}), 4.45(\mathrm{t}, J=7.0 \mathrm{~Hz}$, $2 \mathrm{H}), 3.90(\mathrm{~d}, J=12.3 \mathrm{~Hz}, 6 \mathrm{H}), 3.06(\mathrm{t}, J=7.0 \mathrm{~Hz}, 2 \mathrm{H}) .{ }^{13} \mathrm{C}$ NMR $\left(126 \mathrm{MHz} \mathrm{CDCl}_{3}\right) \delta 167.05(\mathrm{C}-1), 153.15(\mathrm{C}-6), 139.53$ (C-5), 137.99 (C-3), 128.99 (C-3'), 128.64 (C-5', C-7'), 128.52 (C-4', C-8'), 126.55 (C-6'), 124.19 (C-4, C-8), 119.37 (C-9), 119.21 (C-2), 113.96 (C-7), 65.01 (C-1'), $61.35\left(\mathrm{C}-5,-\mathrm{OCH}_{3}\right)$, $55.90\left(\mathrm{C}-6,-\mathrm{OCH}_{3}\right), 35.26\left(\mathrm{C}-2{ }^{\prime}\right)$. MS (ESI) $\mathrm{m} / z: 313[\mathrm{M}+$ $\mathrm{H}]^{+}$, calcd. for $\mathrm{C}_{19} \mathrm{H}_{20} \mathrm{O}_{4} 312.14$.

3-p-tolyl-acrylic acid phenethyl ester (6): White solid, yield $90 \%$, m.p.: 67.4-68.4 ${ }^{\circ} \mathrm{C} ;{ }^{1} \mathrm{H} \mathrm{NMR}\left(500 \mathrm{MHz}, \mathrm{CDCl}_{3}\right) \delta$ $7.68(\mathrm{~d}, J=16.0 \mathrm{~Hz}, 1 \mathrm{H}), 7.45(\mathrm{~d}, J=8.0 \mathrm{~Hz}, 2 \mathrm{H}), 7.36(\mathrm{t}, J=$ $7.4 \mathrm{~Hz}, 2 \mathrm{H}), 7.32-7.26$ (m, 3H), 7.22 (d, J=7.8 Hz, 2H), 6.42 $(\mathrm{d}, J=16.0 \mathrm{~Hz}, 1 \mathrm{H}), 4.45(\mathrm{t}, J=7.1 \mathrm{~Hz}, 2 \mathrm{sH}), 3.05(\mathrm{t}, J=7.0$ $\mathrm{Hz}, 2 \mathrm{H}), 2.41$ (s, 3H). ${ }^{13} \mathrm{C}$ NMR $\left(126 \mathrm{MHz}, \mathrm{CDCl}_{3}\right) \delta 167.10$ (C-1), 144.87 (C-3), 140.72 (C-3'), 137.96 (C-7), 131.71 (C4), 129.63 (C-6,C-8), 128.96 (C-5',C-7'), 128.53 (C-4',C-8'), 128.10 (C-5,C-9), 126.57 (C-6'), 116.98 (C-2), 64.95 (C-1'), 35.26 (C-2'), $21.48\left(\mathrm{C}-7, \mathrm{OCH}_{3}\right)$; MS (ESI) $\mathrm{m} / \mathrm{z}: 267[\mathrm{M}+\mathrm{H}]^{+}$, calcd. for $\mathrm{C}_{18} \mathrm{H}_{18} \mathrm{O}_{2}$ 266.13. 
3-(2-Methoxy-phenyl)acrylic acid phenethyl ester (7): Colourless oil, yield $80 \%,{ }^{1} \mathrm{H}$ NMR $\left(500 \mathrm{MHz}, \mathrm{CDCl}_{3}\right) \delta 8.03$ $(\mathrm{d}, J=16.2 \mathrm{~Hz}, 1 \mathrm{H}), 7.52(\mathrm{t}, J=12.0 \mathrm{~Hz}, 1 \mathrm{H}), 7.40-7.23(\mathrm{~m}$, $6 \mathrm{H}), 7.02-6.90(\mathrm{~m}, 2 \mathrm{H}), 6.55(\mathrm{~d}, J=16.2 \mathrm{~Hz}, 1 \mathrm{H}), 4.45(\mathrm{t}, J=$ $7.1 \mathrm{~Hz}, 2 \mathrm{H}), 3.92(\mathrm{~s}, 3 \mathrm{H}), 3.06(\mathrm{t}, J=7.1 \mathrm{~Hz}, 2 \mathrm{H}) .{ }^{13} \mathrm{C} \mathrm{NMR}$ $\left(126 \mathrm{MHz} \mathrm{CDCl}_{3}\right) \delta 167.41(\mathrm{C}-1), 158.36(\mathrm{C}-4), 140.28(\mathrm{C}-$ 3), 138.03 (C-3'), 131.49 (C-7), 129.00 (C-5', C-7'), 128.90 (C-4', C-8'), 128.51 (C-9), 126.53 (C-6'), 123.44 (C-8), 120.71 (C-4), 118.55 (C-2), 111.17 (C-6), 64.91 (C-1'), 55.50 (C5, $\left.\mathrm{OCH}_{3}\right), 35.29\left(\mathrm{C}-2^{\prime}\right)$; MS (ESI) $m / z: 283[\mathrm{M}+\mathrm{H}]^{+}$, calcd. for $\mathrm{C}_{18} \mathrm{H}_{18} \mathrm{O}_{3} 282.13$.

3-(3-Methoxy-phenyl)acrylic acid phenethyl ester (8): White solid, yield $85 \%$, m.p.: 43.4-44.4 ${ }^{\circ} \mathrm{C}$; ${ }^{1} \mathrm{H}$ NMR $(500$ $\left.\mathrm{MHz}, \mathrm{CDCl}_{3}\right) \delta 7.67(\mathrm{~d}, J=16.0 \mathrm{~Hz}, 1 \mathrm{H}), 7.38-7.25(\mathrm{~m}, 6 \mathrm{H})$, $7.14(\mathrm{~d}, J=7.6 \mathrm{~Hz}, 1 \mathrm{H}), 7.07(\mathrm{~s}, 1 \mathrm{H}), 6.97(\mathrm{dd}, J=8.2,2.0$ $\mathrm{Hz}, 1 \mathrm{H}), 6.45$ (d, $J=16.0 \mathrm{~Hz}, 1 \mathrm{H}), 4.46$ (t, $J=7.1 \mathrm{~Hz}, 2 \mathrm{H})$, $3.86(\mathrm{~s}, 3 \mathrm{H}), 3.06(\mathrm{t}, J=7.1 \mathrm{~Hz}, 2 \mathrm{H}) .{ }^{13} \mathrm{C} \mathrm{NMR}(126 \mathrm{MHz}$, $\left.\mathrm{CDCl}_{3}\right) \delta 166.84(\mathrm{C}-1), 159.92(\mathrm{C}-6), 144.79(\mathrm{C}-3), 137.90$ (C-3'), 135.80 (C-4), 129.90 (C-8), 128.95 (C-5', C-7'), 128.55 (C-4', C-8'), 126.60 (C-6'), 120.82 (C-9), 118.38 (C-2), 116.17 (C-7), 112.98 (C-5), $65.04(\mathrm{C}-1 '), 55.32\left(\mathrm{C}-6, \mathrm{OCH}_{3}\right), 35.23$ (C-2'); MS (ESI) m/z: $283[\mathrm{M}+\mathrm{H}]^{+}$, calcd. for $\mathrm{C}_{18} \mathrm{H}_{18} \mathrm{O}_{3} 282.13$.

3-(2,5-Dimethoxy-phenyl)acrylic acid phenethyl ester (9): Yellow oil, yield $80 \%,{ }^{1} \mathrm{H}$ NMR (500 MHz, DMSO-d 6 ) $\delta$ $8.00(\mathrm{~d}, J=16.1 \mathrm{~Hz}, 1 \mathrm{H}), 7.37-7.24(\mathrm{~m}, 4 \mathrm{H}), 7.07$ (d, $J=2.6$ $\mathrm{Hz}, 1 \mathrm{H}), 6.96-6.85$ (m, 2H), 6.52 (dd, $J=16.1,12.3 \mathrm{~Hz}, 1 \mathrm{H})$, $4.45(\mathrm{t}, J=7.1 \mathrm{~Hz}, 1 \mathrm{H}), 3.82(\mathrm{dd}, J=25.1,17.1 \mathrm{~Hz}, 8 \mathrm{H}), 3.05$ $(\mathrm{t}, J=7.1 \mathrm{~Hz}, 1 \mathrm{H}) .{ }^{13} \mathrm{C}$ NMR $\left(126 \mathrm{MHz}, \mathrm{DMSO}-\mathrm{d}_{6}\right) \delta 167.25$ (C-1), 153.53 (C-8), 152.85 (C-5), 140.01 (C-3), 137.99 (C3'), 128.98 (C-5', C-7'), 128.51 (C-4', C-8'), 126.53 (C-6'), 124.00 (C-4), 118.55 (C-2), 117.14 (C-6), 117.11 (C-7), 113.33 (C-9), $64.93(\mathrm{C}-1 '), 56.11\left(\mathrm{C}-5, \mathrm{OCH}_{3}\right), 51.61\left(\mathrm{C}-8, \mathrm{OCH}_{3}\right)$, $35.27\left(\mathrm{C}-2^{\prime}\right)$; $\mathrm{MS}$ (ESI) m/z: $313[\mathrm{M}+\mathrm{H}]^{+}$, calcd. for $\mathrm{C}_{19} \mathrm{H}_{20} \mathrm{O}_{4}$ 312.14 .

3-(2,3,4-Trimethoxy-phenyl)acrylic acid phenethyl ester (10): White solid, yield $90 \%$, m.p.: $54.3-55.3{ }^{\circ} \mathrm{C}$; ${ }^{1} \mathrm{H}$ NMR (500 MHz, $\left.\mathrm{CDCl}_{3}\right) \delta 7.90(\mathrm{~d}, J=16.1 \mathrm{~Hz}, 1 \mathrm{H}), 7.39$ $7.23(\mathrm{~m}, 6 \mathrm{H}), 6.71(\mathrm{~d}, J=8.8 \mathrm{~Hz}, 1 \mathrm{H}), 6.43(\mathrm{~d}, J=16.1 \mathrm{~Hz}$, $1 \mathrm{H}), 4.44(\mathrm{t}, J=7.1 \mathrm{~Hz}, 2 \mathrm{H}), 3.96-3.86(\mathrm{~m}, 9 \mathrm{H}), 3.05(\mathrm{t}, J=$ $7.1 \mathrm{~Hz}, 2 \mathrm{H}) .{ }^{13} \mathrm{C}$ NMR $\left(126 \mathrm{MHz}, \mathrm{CDCl}_{3}\right) \delta 167.38(\mathrm{C}-1)$, 155.55 (C-5), 153.32 (C-7), 142.40 (C-3), 139.76 (C-3'), 138.06 (C-6), 128.98 (C-5', C-7'), 128.50 (C-4', C-8'), 126.52 (C-6'), 123.13 (C-9), 121.52 (C-2), 116.95 (C-4), 107.63 (C8), $64.85\left(\mathrm{C}-1^{\prime}\right), 61.43\left(\mathrm{C}-5, \mathrm{OCH}_{3}\right), 60.90\left(\mathrm{C}-6, \mathrm{OCH}_{3}\right), 56.08$ $\left(\mathrm{C}-7, \mathrm{OCH}_{3}\right), 35.29\left(\mathrm{C}-2^{\prime}\right)$. MS (ESI) $m / z: 343[\mathrm{M}+\mathrm{H}]^{+}$, calcd. for $\mathrm{C}_{20} \mathrm{H}_{22} \mathrm{O}_{5} 342.15$.

3-(4-Dimethylamino-phenyl)acrylic acid phenethyl ester (11): Yellow solid, yield $85 \%$, m.p.: $117.4-118.4{ }^{\circ} \mathrm{C} ;{ }^{1} \mathrm{H}$ NMR $\left(500 \mathrm{MHz}, \mathrm{CDCl}_{3}\right) \delta 7.65(\mathrm{~d}, J=15.8 \mathrm{~Hz}, 1 \mathrm{H}), 7.45(\mathrm{~d}$, $J=8.7 \mathrm{~Hz}, 2 \mathrm{H}), 7.37-7.23(\mathrm{~m}, 5 \mathrm{H}), 6.71(\mathrm{~d}, J=8.4 \mathrm{~Hz}, 2 \mathrm{H})$, $6.25(\mathrm{~d}, J=15.8 \mathrm{~Hz}, 1 \mathrm{H}), 4.47-4.39(\mathrm{~m}, 2 \mathrm{H}), 3.09-2.98(\mathrm{~m}$, 8H). ${ }^{13} \mathrm{C} \mathrm{NMR}\left(126 \mathrm{MHz}, \mathrm{CDCl}_{3}\right) \delta 167.77(\mathrm{C}-1), 151.73(\mathrm{C}-$ 7), 145.34 (C-3), 138.14 (C-3'), 129.77 (C-5', C-7'), 128.98 (C-4', C-8'), 128.49 (C-5, C-9), 126.49 (C-6'), 112.46 (C-2), 111.91 (C-6, C-8), 64.65 (C-1'), $40.20\left(\mathrm{C}-7, \mathrm{~N}\left(\mathrm{CH}_{3}\right)_{2}\right), 35.35$ (C-2'); MS (ESI) m/z: $296[\mathrm{M}+\mathrm{H}]^{+}$, calcd. for $\mathrm{C}_{19} \mathrm{H}_{21} \mathrm{NO}_{2}$ 295.16.

3-(3-Hydroxy-phenyl)acrylic acid phenethyl ester (12): $500 \mathrm{mg}$ of 3-(3-hydroxy-phenyl)-acrylic acid was dissolved in $6 \mathrm{~mL}$ of 1,3-dimethyl-2-imidazolidinone (DMI). $350 \mathrm{mg}$ exsiccated sodium carbonate $\left(\mathrm{Na}_{2} \mathrm{CO}_{3}\right)$ was added while stirring. Stirring continued for $0.5 \mathrm{~h}$. At the end of this period a solution of $0.43 \mathrm{~mL}$ of phenethyl bromide dissolved in $1 \mathrm{~mL}$ DMI was added slowly to the reaction mixture over a period of $0.5 \mathrm{~h}$. Following the addition, a small amount of potassium iodide (KI) was added to the reaction mixture. This mixture was stirred at room temperature for $9 \mathrm{~h}$ after which the temperature was reduced to $15^{\circ} \mathrm{C}$ and the reaction mixture was stirred for another $12 \mathrm{~h}$. The reaction mixture was slowly added to $30 \mathrm{~mL}$ of ice water while stirring and extracted with ethyl acetate $(3 \times 15 \mathrm{~mL})$. The aqueous layer was acidified by dropwise addition of $1 \mathrm{M}$ aqueous $\mathrm{HCl}$ and extracted with ethyl acetate. The combined organic extract was washed with saturated sodium chloride solution $(3 \times 15 \mathrm{~mL})$. The organic layer was dried over anhydrous $\mathrm{Na}_{2} \mathrm{SO}_{4}$, filtered and concentrated under reduced pressure followed by purification of the crude products by column chromatography, thereby producing 3-(3-hydroxyphenyl)acrylic acid phenethyl ester. White solid, yield $80 \%$, m.p.: $100.8-101.8{ }^{\circ} \mathrm{C} ;{ }^{1} \mathrm{H}$ NMR $\left(500 \mathrm{MHz}, \mathrm{CDCl}_{3}\right) \delta 7.66(\mathrm{~d}$, $J=16.0 \mathrm{~Hz}, 1 \mathrm{H}), 7.32(\mathrm{dt}, J=16.9,7.9 \mathrm{~Hz}, 6 \mathrm{H}), 7.14-7.02(\mathrm{~m}$, $2 \mathrm{H}), 6.92(\mathrm{~d}, J=7.9 \mathrm{~Hz}, 1 \mathrm{H}), 6.43(\mathrm{~d}, J=16.0 \mathrm{~Hz}, 1 \mathrm{H}), 6.01$ $(\mathrm{d}, J=5.8 \mathrm{~Hz}, 1 \mathrm{H}), 4.47(\mathrm{t}, J=7.0 \mathrm{~Hz}, 2 \mathrm{H}), 3.05(\mathrm{t}, J=7.0 \mathrm{~Hz}$, $2 \mathrm{H}) .{ }^{13} \mathrm{C} \mathrm{NMR}\left(126 \mathrm{MHz}, \mathrm{CDCl}_{3}\right) \delta 167.35(\mathrm{C}-1), 156.24(\mathrm{C}-$ 6), 145.00 (C-3), 137.78 (C-3'), 135.84 (C-4), 130.13 (C-8), 128.95 (C-5', C-7'), 128.57 (C-4', C-8'), 126.64 (C-6'), 120.76 (C-9), 118.21 (C-2), 117.67 (C-7), 114.65 (C-5), 65.30 (C-1'), $35.18\left(\mathrm{C}-2^{\prime}\right)$; MS (ESI) $m / z: 269[\mathrm{M}+\mathrm{H}]^{+}$, calcd. for $\mathrm{C}_{17} \mathrm{H}_{16} \mathrm{O}_{3} 268.11$.

Compounds 13-17 were prepared in the same manner as described for compound $\mathbf{1 2}$.

3-(4-Hydroxy-phenyl)acrylic acid phenethyl ester (13): White solid, yield $75 \%$, m.p.: 90.5-91.5 ${ }^{\circ} \mathrm{C} ;{ }^{1} \mathrm{H}$ NMR (500 $\left.\mathrm{MHz}, \mathrm{CDCl}_{3}\right) \delta 7.65(\mathrm{~d}, J=16.0 \mathrm{~Hz}, 1 \mathrm{H}), 7.44(\mathrm{~d}, J=8.4 \mathrm{~Hz}$, 2H), 7.36-7.24 (m, 5H), 6.89 (d, $J=8.5 \mathrm{~Hz}, 2 \mathrm{H}), 6.57-6.38$ $(\mathrm{m}, 1 \mathrm{H}), 6.31(\mathrm{~d}, J=15.9 \mathrm{~Hz}, 1 \mathrm{H}), 4.46(\mathrm{t}, J=7.0 \mathrm{~Hz}, 2 \mathrm{H})$, $3.05(\mathrm{t}, J=7.0 \mathrm{~Hz}, 2 \mathrm{H}) ;{ }^{13} \mathrm{C} \mathrm{NMR}\left(126 \mathrm{MHz}, \mathrm{CDCl}_{3}\right) \delta 167.77$ (C-1), 158.19 (C-7), 144.97 (C-3), 137.89 (C-3'), 130.05 (C-5', C-7'), 128.95 (C-4', C-8'), 128.55 (C-5, C-9), 126.94 (C-4), 126.60 (C-6'), 115.96 (C-2), 115.15 (C-6, C-8), 65.09 (C-1'), $35.23\left(\mathrm{C}-2^{\prime}\right)$; MS (ESI) $m / z: 268.9[\mathrm{M}+\mathrm{H}]^{+}$, calcd. for $\mathrm{C}_{17} \mathrm{H}_{16} \mathrm{O}_{3} 268.11$.

3-(4-Hydroxy-3-methoxy-phenyl)acrylic acid phenethyl ester (14): Colourless oil, yield $70 \%$; ${ }^{1} \mathrm{H}$ NMR (500 MHz, $\left.\mathrm{CDCl}_{3}\right) \delta 7.63(\mathrm{~d}, J=15.9 \mathrm{~Hz}, 1 \mathrm{H}), 7.35(\mathrm{t}, J=7.4 \mathrm{~Hz}, 2 \mathrm{H})$, 7.28 (dd, $J=14.6,7.7 \mathrm{~Hz}, 3 \mathrm{H}), 7.10-7.03(\mathrm{~m}, 2 \mathrm{H}), 6.95$ (d, $J=8.1 \mathrm{~Hz}, 1 \mathrm{H}), 6.31(\mathrm{~d}, J=15.9 \mathrm{~Hz}, 1 \mathrm{H}), 5.95(\mathrm{~s}, 1 \mathrm{H}), 4.45$ $(\mathrm{t}, J=7.1 \mathrm{~Hz}, 2 \mathrm{H}), 3.95(\mathrm{~s}, 3 \mathrm{H}), 3.05(\mathrm{t}, J=7.0 \mathrm{~Hz}, 2 \mathrm{H}) .{ }^{13} \mathrm{C}$ NMR $\left(126 \mathrm{MHz} \mathrm{CDCl}_{3}\right) \delta 167.20(\mathrm{C}-1), 148.02(\mathrm{C}-6), 146.80$ (C-3), 144.96 (C-7), 137.97 (C-3'), 128.94 (C-4), 128.53 (C5', C-7'), 127.01 (C-4', C-8'), 126.56 (C-6'), 123.12 (C-9), 115.44 (C-2), 114.74 (C-8), 109.37 (C-5), 64.88 (C-1'), 55.97 $\left(\mathrm{C}-6, \mathrm{OCH}_{3}\right), 35.26(\mathrm{C}-2 \mathrm{\prime})$; MS (ESI) $\mathrm{m} / z: 298.9[\mathrm{M}+\mathrm{H}]^{+}$, calcd. for $\mathrm{C}_{18} \mathrm{H}_{18} \mathrm{O}_{4} 298.12$.

3-(2-Hydroxy-phenyl)acrylic acid phenethyl ester (15): White solid, yield $75 \%$, m.p.: 102.1-103.1 ${ }^{\circ} \mathrm{C}$; ${ }^{1} \mathrm{H}$ NMR (500 $\left.\mathrm{MHz}, \mathrm{CDCl}_{3}\right) \delta 8.10(\mathrm{~d}, J=16.2 \mathrm{~Hz}, 1 \mathrm{H}), 7.49(\mathrm{~d}, J=7.7 \mathrm{~Hz}$, 1H), 7.39-7.24 (m, 6H), 7.02-6.84 (m, 3H), 6.68 (d, $J=16.2$ $\mathrm{Hz}, 1 \mathrm{H}), 4.49$ (t, $J=7.1 \mathrm{~Hz}, 2 \mathrm{H}), 3.07(\mathrm{t}, J=7.1 \mathrm{~Hz}, 2 \mathrm{H}) .{ }^{13} \mathrm{C}$ 
TABLE-1

$\mathrm{IC}_{50}$ VALUES OF SYNTHETIC CAFFEIC ACID PHSENETHYL ESTER ANALOGUES AGAINST BEL-7402 AND MCF-7 ( $\left.\mu \mathrm{M}\right)$

\begin{tabular}{ccccccccc}
\hline NO. & $1-11$ & 12 & 13 & 14 & 15 & 16 & 17 & CAPE \\
\hline Bel-7402 & $>200$ & 51.36 & 55.67 & 41.87 & 44.59 & 80.13 & 81.26 & 34.6 \\
MCF-7 & $>200$ & 67.11 & 92.87 & 51.98 & 58.55 & 101.60 & $>200$ & 42.26 \\
\hline
\end{tabular}

NMR (126 MHz, $\left.\mathrm{CDCl}_{3}\right) \delta 168.50(\mathrm{C}-1), 155.51$ (C-5), 141.02 (C-3), 137.86 (C-3'), 131.54 (C-7), 129.24 (C-5', C-7'), 128.98 (C-9), 128.57 (C-4', C-8'), $126.62\left(\mathrm{C}-66^{\prime}\right), 121.70(\mathrm{C}-4), 120.71$ (C-8), 118.12 (C-2), 116.48 (C-6), $65.27\left(\mathrm{C}^{\prime} \mathbf{1}^{\prime}\right), 35.23$ (C-2'); MS (ESI) $m / z: 269[\mathrm{M}+\mathrm{H}]^{+}$, calcd. for $\mathrm{C}_{17} \mathrm{H}_{16} \mathrm{O}_{3} 268.11$.

4-Hydroxy-3-methoxy-benzoic acid phenethyl ester (16): Colourless oil, yield $70 \%,{ }^{1} \mathrm{H}$ NMR $\left(500 \mathrm{MHz}, \mathrm{CDCl}_{3}\right)$ $\delta 7.65(\mathrm{dd}, J=8.3,1.5 \mathrm{~Hz}, 1 \mathrm{H}), 7.54(\mathrm{~d}, J=1.3 \mathrm{~Hz}, 1 \mathrm{H}), 7.41$ $7.23(\mathrm{~m}, 5 \mathrm{H}), 6.96(\mathrm{~d}, J=8.3 \mathrm{~Hz}, 1 \mathrm{H}), 6.13(\mathrm{~d}, J=4.6 \mathrm{~Hz}$, $1 \mathrm{H}), 4.54(\mathrm{t}, J=7.0 \mathrm{~Hz}, 2 \mathrm{H}), 3.94(\mathrm{~s}, 3 \mathrm{H}), 3.10(\mathrm{t}, J=7.0 \mathrm{~Hz}$, $2 \mathrm{H}) ;{ }^{13} \mathrm{C}$ NMR $\left(126 \mathrm{MHz}, \mathrm{CDCl}_{3}\right) \delta 166.31(\mathrm{C}-1), 150.05(\mathrm{C}-$ 4), 146.17 (C-5), 138.05 (C-3'), 129.01 (C-5',C-7'), 128.53 (C-4', C-8'), 126.58 (C-6'), 124.18 (C-2), 122.41 (C-7), 114.10 (C-3), 111.77 (C-6), 65.32 (C-1'), 56.07 (C-4, $\left.\mathrm{OCH}_{3}\right), 35.33$ (C-2'); MS (ESI) $m / z: 273[\mathrm{M}+\mathrm{H}]^{+}$, calcd. for $\mathrm{C}_{16} \mathrm{H}_{16} \mathrm{O}_{4} 272.10$.

3-Hydroxy-benzoic acid phenethyl ester (17): White solid, yield $80 \%$, m.p.: 78.4-79.1 ${ }^{\circ} \mathrm{C}$; ${ }^{1} \mathrm{H}$ NMR $(500 \mathrm{MHz}$, $\left.\mathrm{CDCl}_{3}\right) \delta 7.65-7.55(\mathrm{~m}, 2 \mathrm{H}), 7.42-7.20(\mathrm{~m}, 6 \mathrm{H}), 7.10(\mathrm{dd}, J=$ $8.1,1.3 \mathrm{~Hz}, 1 \mathrm{H}), 6.12(\mathrm{~s}, 1 \mathrm{H}), 4.56(\mathrm{t}, J=7.0 \mathrm{~Hz}, 2 \mathrm{H}), 3.10(\mathrm{t}$, $J=6.9 \mathrm{~Hz}, 2 \mathrm{H}) ;{ }^{13} \mathrm{C} \mathrm{NMR}\left(126 \mathrm{MHz}, \mathrm{CDCl}_{3}\right) \delta 166.79(\mathrm{C}-1)$, 155.93 (C-4), 137.76 (C-3'), 131.49 (C-2), 129.74 (C-6), 128.97 (C-5', C-7'), 128.59 (C-4', C-8'), 126.66 (C-6'), 121.89 (C-7), 120.37 (C-5), 116.39 (C-3), 65.82 (C-1'), 35.18 (C-2'); MS (ESI) $m / z: 243[\mathrm{M}+\mathrm{H}]^{+}$, calcd. for $\mathrm{C}_{15} \mathrm{H}_{14} \mathrm{O}_{3} 242.09$.

Biological evaluation: Bel-7402 and MCF-7 cells were cultured in DMEM containing $10 \%(\mathrm{v} / \mathrm{v})$ fetal bovine serum (HyClone, Logan, UT, USA), $1 \%$ penicillin and $1 \%$ streptomycin at a humidified atmosphere with $5 \% \mathrm{CO}_{2}$ at $37^{\circ} \mathrm{C}$. The cells were plated in 96 well microplates at a density of $2 \times 10^{4}$ cells $/ \mathrm{mL}$ ( $100 \mu \mathrm{l}$ per well). After $24 \mathrm{~h}$ of incubation to allow for cell attachment, different concentrations of compounds 1-17 were added and stock solutions of the synthesized compounds $(10 \mathrm{mmol} / \mathrm{mL})$ were prepared by dissolving them in DMSO. Appropriate dilutions of the synthesized compounds were prepared in growth medium and added to the cells. The viable cell growth after incubation for $72 \mathrm{~h}$ with synthesized compounds was determined using the MTT assay ${ }^{16}$. The absorbance was determined at $490 \mathrm{~nm}$ using a microplate reader.

\section{RESULTS AND DISCUSSION}

The synthetic routes for the desired compounds 1-11 and 12-17 are outlined in Scheme-I. Caffeic acid phenethyl ester (CAPE) has been synthesized previously by several steps ${ }^{1,14,17,18}$. Among those synthetic methods, we selected one-pot esterification of caffeic acid with (2-bromo-ethyl)benzene in dichloromethane $\left(\mathrm{CH}_{2} \mathrm{Cl}_{2}\right)$ to prepare compounds $\mathbf{1 2 - 1 7 ^ { 1 4 }}$. Compounds 1-11 were prepared by conventional procedures as following.

Compounds 12-17 with a hydroxy group on the benzene ring made the structure more unstable compared with compounds 1-11. Therefore, the yields of compounds 12-17 (about $75 \%$ ) were lower than that of compounds 1-11 (about $90 \%$ ). In the course of obtaining 12-17, HMPA which was used in literature was replaced by DMI because of its carcinogenicity $^{14,19}$. Besides the low toxicity of DMI, it was a cheap and a good aprotic polar solvent. Moreover, the temperature $\left(50-60{ }^{\circ} \mathrm{C}\right)$ was a critical factor to the experiment and at room temperature, 12-17 were not obtained.

Biological activities: Caffeic acid phenethyl ester together with its seventeen analogues (1-17) were tested for their cytotoxicities activities against two different human cancer cell lines, that is, Bel-7402 and MCF-7. The antiproliferative activities of 1-17 are summarized in terms of their $\mathrm{IC}_{50}$ values in Table-1.

Interestingly, the CAPE analogues possessed similar strength of activity toward the two tested cell lines. The order of their $\mathrm{IC}_{50}$ values was CAPE $<14<15<12<13<16<17$ $\leq 1-11$.

Compound 14 showed stronger antiproliferative activity than other analogues. The inhibitory activities of compound 12 (with $m$-hydroxy-phenyl) against two cell lines are stronger than that of compound $\mathbf{1 3}$ (with $p$-hydroxy-phenyl) while are weaker than that of compound $\mathbf{1 5}$ (with $o$-hydroxy-phenyl). This indicated that the methoxy groups on the phenyl ring may enhance antiproliferative activity on condition that the ring had the adjacent hydroxyl.

Comparison of compounds 1-11 and compounds 12-15 revealed that 12-15 possessed stronger activity against the two tested cell lines. On the other hand, the effects of 16 and 17 on Bel-7402 were obvious but on MCF-7 were significantly less. The results indicated that the presence of both phenolic hydroxyl groups and $\alpha, \beta$-unsaturated double bond could be playing an important role in their antiproliferative activities.

\section{Conclusion}

This study investigated the structure-activity relationship of CAPE and its analogues. Seventeen caffeic acid phenethyl ester analogues were prepared in order to test their antiproliferation activities on the two cells. The simultaneous cytotoxicity evaluation on the two cells of compounds 1-17 provides valuable information regarding the prospective lead compounds.

Among those, CAPE and compounds 12, 13, 14, 15, 16, 17 presented obvious antitumor activities against Bel-7402 with $\mathrm{IC}_{50}(\mu \mathrm{M})$ values $(51.36,55.67,41.87,44.59,80.13$, 81.26 ) and that CAPE and compounds $\mathbf{1 2}, \mathbf{1 3}, \mathbf{1 4}, 15$ possessed strong antitumor activities against MCF-7 with the values $(42.26,67.11,92.87,51.98,58.55)$. The results indicated that compounds with phenolic hydroxyl groups and $\alpha, \beta$-unsaturated double bond exhibited greater inhibitory activities on the two cancer cell lines than those compounds without them. The methoxy groups on the phenyl ring and the location of aromatic hydroxyl groups were critical factors for the compounds' activities.

\section{ACKNOWLEDGEMENTS}

This part of work is partially supported by the National Natural Science Foundation of China (No.81073017; No. 
81173519) and the Innovation Team Project Foundation of Beijing University of Chinese Medicine named 'Lead Compounds Discovering and Developing Innovation Team Project Foundation' (No. 2011-CXTD-15).

\section{REFERENCES}

1. D. Grunberger, R. Banerjee, K. Eisinger, E.M. Oltz, L. Efros, M. Caldwell, V. Estevez and K. Nakanishi, Experientia, 44, 230 (1988).

2. B.H. Ackerman, R.E. Guilday, C.L. Reigart, M.L. Patton and L.R. Haith, J. Burn Care Res., 34, 1 (2013).

3. S. Akyol, G. Ozturk, Z. Ginis, F. Armutcu, M.R. Yigitoglu and O. Akyol, Nutr. Cancer, 65, 515 (2013).

4. J.H. Park, J.K. Lee, H.S. Kim, S.T. Chung, J.H. Eom, K.A. Kim, S.J. Chung, S.Y. Paik and H.Y. Oh, Int. Immunopharmacol., 4, 429 (2004).

5. H. Göçer and I. Gülçin, Int. J. Food Sci. Nutr., 62, 821 (2011).

6. J. Wu, C. Omene, J. Karkoszka, M. Bosland, J. Eckard, C.B. Klein and K. Frenkel, Cancer Lett., 308, 43 (2011).

7. K.W. Lee, N.J. Kang, J.H. Kim, K.M. Lee, D.E. Lee, H.J. Hur and H.J. Lee, J. Genes Nutr., 2, 319 (2008).

8. Y.-Y. Kuo, H.-P. Lin, C. Huo, L.-C. Su, J. Yang, P.-H. Hsiao, H.-C. Chiang, C.-J. Chung, H.-D. Wang, J.-Y. Chang, Y.-W. Chen and C.-P. Chuu, Int. J. Mol. Sci., 14, 8801 (2013).
9. P. Onori, S. DeMorrow, E. Gaudio, A. Franchitto, R. Mancinelli, J. Venter, S. Kopriva, Y. Ueno, D. Alvaro, J. Savage, G. Alpini, H. Francis, Int. J. Cancer., 125, 565 (2009).

10. J. Lin, D. Grunberger and P.B. Fisher, Cell. Mol. Biol., 38, 513 (1992).

11. H.-P. Lin, C.-Y. Lin, C.-C. Liu, L.-C. Su, C. Huo, Y.-Y. Kuo, J.-C. Tseng, J.-M. Hsu, C.-K. Chen and C.-P. Chuu, Int. J. Mol. Sci., 14, 5264 (2013).

12. M.F. Chen, C.T. Wu, Y.J. Chen, P.C. Keng and W.-C. Chen, J. Radiat. Res. (Tokyo), 45, 253 (2004).

13. T. Nagaoka, A.H. Banskota, Y. Tezuka, I. Saiki and S. Kadota, Bioorg. Med. Chem., 10, 3351 (2002).

14. J.Y. Liu and Y.S. Du, and Westfield, US Patent 20110046410 (2011).

15. J.R. Macías-Pérez, O. Beltrán-Ramírez, V.R. Vásquez-Garzón, M.E. Salcido-Neyoy, P.A. Martínez-Soriano, M.B. Ruiz-Sánchez, E. Ángeles and S. Villa-Treviño, Anticancer Drugs, 24, 394 (2013).

16. T. Mosmann, J. Immunol. Methods, 65, 55 (1983).

17. O.K. Mirzoeva, G.F. Sud'ina, M.A. Pushkareva, G.A. Korshunova, N.V. Sumbatian and S.D. Varfolomeev, Bioorg. Khim., 21, 143 (1995).

18. T.R.J. Burke, M. Fesen, A. Mazumder, J. Yung, J. Wang, A.M. Carothers, D. Grunberger, J. Driscoll, Y. Pommier and K. Kohn, Med. Chem., 38, 4171 (1995).

19. C.-C. Lo and P.-M. Chao, J. Chem. Ecol., 16, 3245 (1990). 